\title{
Postoperative rectal anastomotic complications
}

\author{
Polanecky $\mathrm{O}^{1}$, Adamek $\mathrm{S}^{1}$, Sedy $\mathrm{J}^{2}$, Skorepa $\mathrm{J}^{1}$, Hladik $\mathrm{P}^{1}$, Smejkal $\mathrm{M}^{1}$, Pafko $\mathrm{P}^{1}$, Lischke $\mathrm{R}^{1}$ \\ Third Department of Surgery, First Faculty of Medicine and Teaching Hospital Motol, Prague, \\ Czech Republic.sadamek@post.cz
}

\begin{abstract}
Colorectal cancer represents the most common tumour of the gastrointestinal tract and the second most common tumour in men as well as women. The trend of increasing incidence of colorectal cancer is alerting. We undertook a retrospective study on 588 patients with rectal cancer and operated by rectal resection with anastomosis between the years 2002-2012. In our sample, we observed 54 (9.2\%) cases of anastomosis insufficiencies requiring reoperation. Out of 54 insufficient anastomoses, 36 (66 \%) were in the lower two thirds of the rectum and only $18(34 \%)$ in the oral one. Although we have observed similar occurrences of anastomosis insufficiency in both groups - classical vs. staple suture ( $9.5 \%$ and $9.0 \%$, respectively), the majority of stapler anastomoses (94\%) were made in the aboral part of the rectum. However, we can state that a majority of authors prefer the staple anastomosis as the one with lowest risk, mainly in the distal region of anastomosis. The high ligation of inferior mesenteric artery was performed in $182(31 \%)$ patients; out of these, we observed anastomosis insufficiency in 12 cases (22 \%), which is exactly similar to that in the group of patients without high ligation of the inferior mesenteric artery. We did not observe the use of antibiotics in therapeutical doses as a positive factor for anastomosis insufficiencies, and neither was oncological therapy observed as a risk factor. In our group of patients we agreed that age, level of anastomosis and corticosteroids are high-risk factors.

The purpose of these reports, is for the sake of future to share and reference our experiences with cases of rectal and rectosigmoideal resection over the last 11 years. We consider it important to reference our results, especially the risk factors regarding the healing of rectal anastomosis, because anastomotic healing is a surgical problem with potentially deadly consequences for patients (Tab. 4, Ref. 24). Text in PDF www.elis.sk.

Key words: rectum, anastomosis, complication, human.
\end{abstract}

Colorectal cancer represents the most common tumour of the gastrointestinal tract and the second most common tumour in both men and women. The trend of increasing incidences of the colorectal cancer is alerting. The Czech Republic is the country with highest incidence of colorectal cancer worldwide $(94.4 / 100,000)$. Approximately $55 \%$ of colorectal cancer cases appear in the rectal and rectosigmoideal region. Despite continuous progress in the oncological treatment, the surgery is still the only curative method $(1,2)$. Technical performance and operative techniques are currently standardized. In this study, we present our experience with a sample of patients operated on in the past 11 years, focusing on the technique of anastomosis and its risk factors. In indicated cases ( $78 \%$ of patients), we used neoadjuvant therapy.

${ }^{1}$ Third Department of Surgery, First Faculty of Medicine and Teaching Hospital Motol, Prague, Czech Republic, and ${ }^{2}$ Institute of Physiology, ASCR, Prague, Czech Republic

Address for correspondence: $\mathrm{S}$. Adamek, MD, PhD, Third Department of Surgery, First Faculty of Medicine and Teaching Hospital Motol, V Uvalu 84, CZ-150 06 Prague 5, Czech Republic.

Phone: +420.224438000

Acknowledgement: Supported by the project (Ministry of Health, Czech Republic) for conceptual development of research organization 00064203 (University Hospital Motol, Prague, Czech Republic).

\section{Materials and methods}

\section{Study and patients}

We undertook a retrospective study of 588 patients with rectosigmoideal cancer, in whom rectal resection with anastomosis was performed between years 2002-2012.

\section{Sample}

In years 2002-2012, we operated together on 588 patients, of whom 358 (61\%) were males and 230 (39\%) females. The mean age of patients was $63.53 \pm 6.24$ (males $62.86 \pm 5.98$; females 64.57 \pm 6.35 ; the difference not statistically significant).

\section{Statistical analysis}

The statistical significance between groups was compared using the non-paired Student's t-test $(\mathrm{p}<0.05)$.

\section{Preoperative care}

Before surgery, patients underwent bowel preparation, usually orthograde in combination with clysma.

\section{Surgery}

In our sample, we made lower anterior rectal resection with partial or total mesorectal excision, regional lymphadenectomy 
from iliac vessels to bifurcation of the aorta, and often even to the inferior mesenteric artery, which was not ligated in many cases. Orally, the line of resection was approximately $15 \mathrm{~cm}$ above the tumour, and aborally $1 \mathrm{~cm}$ at minimum below the tumour; in cases where we were close to $1 \mathrm{~cm}$ border, we used peroperative histology. Simultaneous use of operative rectoscope as well as pouche construction is not a routine technique at our department.

\section{Non-surgical treatment}

Since year 2003, we have been using the neoadjuvant chemoradiotherapy or isolated radiotherapy. The unambiguously predominant method was concomitant chemoradiotherapy, mostly XELODA, and the use of 45 Gy radiotherapy, lasting 6-8 weeks. The isolated radiotherapy was used only in some cases, namely when patients were not able to accept chemotherapy. Neoadjuvant therapy was performed in all patients with staging higher than T2N0. In 4 cases, the disease was generalized and a specialist in oncology indicated only palliative treatment. In 49 patients $(9$ $\%$ ) with bowel obstruction, we performed axial sigmoideostomia before neoadjuvant therapy, which was interiorised during subsequent resection. We do not perform protective ileo- or colostomia as standard care, but we did it in only 7 patients due to technical problems during anastomosis construction. During the examined period, we have changed the anastomotic technique. Since 2004, we have been using the transanal stapler anastomosis technique. We mainly use the double stapling method with interruption of rectum by the linear stapler Contour. In $27 \%$ of cases, we used the method of single stapling with the closure of the rectal stub by single sutures. In relation to the use of staplers and indications of neoadjuvant oncological treatment, a decrease in number of rectal amputations is apparent in our sample (Tab. 1). The still used manual technique of anastomosis is based on a connection with single sutures or continuous one in one layer. In cases of technical problems, we perform the test of hermetic closure by transanally instilated solution; we have but repeatedly observed that postoperative hermetic closure does not correlate with postoperative sufficiency in anastomosis.

\section{Postoperative care}

The postoperative care was standardized. Until the postoperative days $2-4$, the patients had only liquid diet, next day they were given mash diet, followed by non-residual diet modified according to clinical status of individual patients and their bowel function.

\section{Risk factors}

The tested risk factors included the level of anastomosis, technique of anastomosis, perioperative blood loss, use of antibiotics, oncological therapy, immunosupression (corticosteroids administration), high ligation of inferior mesenteric artery, and age.

\section{Level of anastomosis}

In our sample, we analysed the role of anastomosis level in anastomosis insufficiency. We divided patients into two groups: (i) oral third (distance of $8-13 \mathrm{~cm}$ from anal verge) and aboral two thirds of rectum (distance of $0-7 \mathrm{~cm}$ from anal verge).

\section{Results}

\section{Patients}

Out of our sample of 588 patients, we have observed 54 patients $(9 \%)$ with insufficiency, while 40 of them (74\%) were older than 60 years. Mean age of patients with insufficiency was but not statistically significant from other patients $(64.25 \pm 6.12 \mathrm{vs} 62.14$ $\pm 5.96 ; \mathrm{p}=0.34)$. The level of anastomosis in patients with insufficient anastomosis was (i) $0-7 \mathrm{~cm}$ from anus in 36 patients $(67 \%)$ and (ii) $8-13 \mathrm{~cm}$ from anus in 18 patients $(33 \%)$.

\section{The technique of anastomosis}

Together, we have performed 178 anastomoses with manual method, of which $17(10 \%)$ were insufficient and 410 stapler anastomoses, of which 37 (9\%) were insufficient. Thus, out of our sample of 588 patients, together 410 patients $(70 \%)$ underwent stapler anastomosis. Out of the sample of 54 patients with insufficiency, 37 patients (69\%) had stapler anastomosis, whereas out of the rest of 534 of patients from the sample, 373 (70\%) of patients had stapler anastomosis. The difference between these groups was not statistically significant (Student's t-test, $\mathrm{p}=0.83$ ). Thus, the type of the anastomosis had no predictive value on the occurrence of anastomosis insufficiency.

\section{Reoperation}

As a criterion for anastomosis insufficiency, we have used the indication for reoperation on the basis of the clinical status of the patient (stercoral secretion from drainage, signs of peritonitis, increased temperature, increase in inflammatory markers), or signs of anastomosis insufficiency proved by extralumination of contrast on CT or irigographic examination. The numbers of patients who underwent rectal resection and patients with anastomosis insuffi-

Tab. 1. Overview of numbers of patients with colorectal cancer, treated in our department between years 2002-2012.

\begin{tabular}{lccccccccccc}
\hline Year & 2002 & 2003 & 2004 & 2005 & 2006 & 2007 & 2008 & 2009 & 2010 & 2011 & 2012 \\
\hline Rectal Resection & 51 & 57 & 60 & 62 & 61 & 58 & 59 & 42 & 47 & 48 & 43 \\
Colorectal Resection & 111 & 126 & 132 & 142 & 143 & 139 & 141 & 114 & 131 & 142 & 138 \\
Rectal Amputation & 11 & 17 & 8 & 8 & 9 & 8 & 9 & 7 & 6 & 7 & 8 \\
\hline
\end{tabular}

Tab. 2. Numbers of patients who underwent rectal resection and patients with anastomosis insufficiency, who were reoperated.

\begin{tabular}{lcccccccccccc}
\hline Year & 2002 & 2003 & 2004 & 2005 & 2006 & 2007 & 2008 & 2009 & 2010 & 2011 & 2012 \\
\hline Rectal resection & 51 & 57 & 60 & 62 & 61 & 58 & 59 & 42 & 47 & 48 & 43 & 5 \\
Anastomosis insufficiency & 4 & 4 & 5 & 6 & 4 & 5 & 3 & 3 & 5 & 7 & 8 \\
\hline
\end{tabular}


Tab. 3. Colorectal anastomotic dehiscence in relation to the level of anastomosis.

\begin{tabular}{|c|c|c|c|c|c|c|}
\hline Authors & $\mathrm{n}$ & Year & Level of anastomosis & Leak rate & Level of anastomosis & Leak rate \\
\hline Goligher et al. & 73 & 1970 & extraperitoneal & $69 \%$ & intraperitoneal & $40 \%$ \\
\hline Shrock et al. & 339 & 1973 & extraperitoneal & $11 \%$ & intraperitoneal & $6 \%$ \\
\hline Manson et al. & 152 & 1976 & extraperitoneal & $24 \%$ & intraperitoneal & $4 \%$ \\
\hline Heald et al. & 100 & 1981 & $0-7 \mathrm{~cm}^{*}$ & $19 \%$ & $7-15 \mathrm{~cm}^{*}$ & $0 \%$ \\
\hline Heberer et al. & 157 & 1982 & $0-10 \mathrm{~cm}^{*}$ & $20 \%$ & $10-15 \mathrm{~cm}^{*}$ & $14 \%$ \\
\hline Vignali et al. & 1014 & 1997 & $0-7 \mathrm{~cm}^{*}$ & $8 \%$ & $7-15 \mathrm{~cm}^{*}$ & $1 \%$ \\
\hline Rullier et al. & 272 & 1998 & $0-5 \mathrm{~cm}^{*}$ & $19 \%$ & $5-15 \mathrm{~cm}^{*}$ & $5 \%$ \\
\hline
\end{tabular}

*distance from anal vedge

Tab. 4. Comparison of occurence of anastomosis insufficiency between group of patients sutured by classical suture when compared with patients where stapler was used.

\begin{tabular}{lcc}
\hline & Classical suture & Stapler \\
\hline Total number of anastomoses & $178(100 \%)$ & $410(100 \%)$ \\
Anastomoses in aboral part & $13(6 \%)$ & $385(94 \%)$ \\
Insufficiency in anastomosis & $17(9.5 \%)$ & $37(9.0 \%)$ \\
\hline
\end{tabular}

ciency, who were reoperated, is shown in Table 2. In our sample, we observed that $9.2 \%$ of patients needed reoperation due to insufficiency in anastomosis.

\section{Level of anastomosis}

Of all 54 insufficient anastomoses from 2002 to 2012, 36 $(66 \%)$ were in the lower two thirds of rectum and 18 (34\%) were anastomosis in the oral third of rectum. The relation between the level of anastomosis and insufficiency is apparent (Tab. 3).

\section{Type of suture}

The next criterion was the comparison of anastomosis insufficiency occurrence in patients in whom the suture was made by stapler or with manual sutures. Although we have observed similar occurrence of anastomosis insufficiency between both groups (9.5\% vs $9.0 \%)$, the majority of stapler anastomoses (94\%) were made in the aboral part of the rectum, when compared with only $6 \%$ done with classical anastomoses (Tab. 4). In all anastomoses sutured by classical method, monofil PDS in one layer was used. In stapler anastomoses, we did not observe a significant difference between single and double stapling methods. However, albeit more expensive, the double stapling method is easier to perform.

\section{Blood loss}

Out of our sample of 588 patients, together in 165 patients (28\%), the blood loss was higher than $1000 \mathrm{ml}$. Out of the sample of 54 patients with anastomosis insufficiency, 33 patients (61\%) had blood loss higher than 1 liter, whereas of the rest of 534 of patients from the sample, $132(25 \%)$ had blood loss higher than 1 liter. The difference between these groups was statistically significant (Student's t-test, $\mathrm{p}=0.000002$ ). Thus, we can conclude that marked blood loss increases the risk of anastomosis insufficiency.

\section{High ligature of inferior mesenteric artery}

Out of our sample of 588 patients, together 182 patients (31\%) underwent high ligature of inferior mesenteric artery. Of the sample of 54 patients with insufficiency, 12 patients ( $22 \%$ ) had high ligature of inferior mesenteric artery, whereas out of the rest of 534 of patients from the sample, $170(32 \%)$ had high ligature of inferior mesenteric artery. The difference between these groups was not statistically significant (Student's T-test, $\mathrm{p}=0.15$ ). Thus, although the data indicate that high ligature of inferior mesenteric artery might slightly decrease the occurrence of anastomosis insufficiency, the differences are not statistically relevant.

\section{Antibiotics}

The prophylactic use of antibiotics (one shot) was a standard care in our sample. During postoperative course, out of our sample of 588 patients, together 86 patients $(15 \%)$ required antibiotics in therapeutic doses. Out of the sample of 54 patients with insufficiency, 22 patients $(41 \%)$ had antibiotics in therapeutic doses, whereas out of the rest of 534 patients from the sample, 64 (12\%) had antibiotics. The difference between these groups was statistically significant (Student's t-test, $\mathrm{p}=0.000000008$ ). Thus, we can conclude that patients with anastomosis insufficiency required antibiotics significantly more often.

\section{Neoadjuvant chemoradiotherapy}

Out of our sample of 588 patients, together 458 patients (78\%) underwent neoadjuvant chemoradiotherapy. Out of the sample of 54 patients with insufficiency, 39 patients ( $72 \%$ ) had neoadjuvant chemoradiotherapy, whereas out of the rest of 534 of patients from the sample, 419 (78 \%) had neoadjuvant chemoradiotherapy. The difference between these groups was not statistically significant (Student's t-test, $\mathrm{p}=0.29$ ). Thus, although the data indicate that neoadjuvant chemoradiotherapy might slightly decrease the occurrence of anastomosis insufficiency, the differences are not statistically relevant.

\section{Corticotherapy (Immunosupresion)}

Out of our sample of 588 patients, together 44 patients (7\%) underwent immunosupresive therapy in form of corticoids. Out of the sample of 54 patients with insufficiency, 8 patients $(15 \%)$ had corticotherapy, whereas out of the rest of 534 of patients from the sample, only 36 (7\%) had immunosupressive therapy by corticoids. The difference between these groups was statistically significant (Student's t-test, $\mathrm{p}=0.03$ ). Thus, we can conclude that corticotherapy increases the risk of anastomosis insufficiency.

In summary, important risk factors for the development of anastomosis insufficiency include the age of patient, level of the anatomosis, corticotherapy and perioperative blood loss. On the other hand, speculative risk factors include inferior mesenteric 
artery ligation, antibiotic administration, oncological therapy and technique of anastomosis performed. In case of the technique of anastomosis, several other factors play a role, such as preferences and experience of the surgeon, and price.

\section{Discussion}

The role of particular risk factors in the development of anastomosis insufficiency is not completely clear. While some are generally accepted as proved risk factors, there are many others, such as gender or smoking, where the risk has not been proved. Nevertheless, the majority of studies show the basic principles of anastomosis construction. It must be sutured without tension and must have vital and well-vascularised borders. Moreover, the preparation must be adequately meticulous, without mechanical injury to the bowel. No distal obstruction should be present.

When we focus on the healing of anastomosis, the important factors are the production of collagen by fibroblasts as well as the proliferation of smooth muscle cells 1 . When intraabdominal infection, such as peritonitis, is present, the synthesis of collagen is inhibited (2), and this can cause important problems hindering the healing of the anastomosis. Significant changes in healing of anastomosis are also described in cases where corticosteroids were administered $(3,24)$, as well as in cases with significant blood loss, requiring blood transfusion $(4,5)$.

The particular anastomosis technique is a topic of discussion. The basic principles are mentioned above. The role of the type of the suture and the number of layers is a question of personal preferences and knowledge of a particular surgeon. There are papers which show better results in two-layered anastomosis (6, 7 ), whereas others found similar results in one-layered and twolayered anastomoses $(8,21)$. The breakthrough in the technique of anastomosis was the development of stapler anastomosis. While in our study, the result is slightly better with the use of stapler anastomosis, there are papers showing similar results of stapler technique and classical suture $(9,10,22)$. On the contrary, other papers proved better results in favour of the stapler anastomosis $(11,12,23)$. It is important to notice that stapler anastomoses are used predominantly in more risky cases, i.e. aboral parts of the rectum. Table 3 summarises the colorectal anastomotic dehiscence in relation to the level of anastomosis. It is evident, that in aboral levels there is a higher incidence of anastomosis insufficiency.

Our results indicate that the use of staplers together with neoadjuvant oncologic therapy significantly improved the number of resections with continence. Another advantage is the shortened time of operation. In conclusion, stapler anastomoses represent a significant improvement in the field of rectal surgery.

The positive impacts of bowel preparation, small pelvic drainage or supportive stomia have been discussed for a few years. Many papers published equal results of insufficiency in anastomosis in patients, who had bowel preparation and those who did not $(13,14)$. The same was published for the local bowel preparation with antibiotics 15 . We do not perform temporary colostomy or ileostomy as standard care, only in high-risk anastomoses, as for example during technical problems in the course of construc- tion of anastomosis. However, many publications present equal results in patients with and without protective stomia $(16,17,20)$.

There are many known risk factors. Verified risk factors include, for example, the age of patient, anastomosis level, peritonitis or corticotherapy; while the suspected risk factors include smoking or male gender. We did not prove that ligation of the inferior mesenteric artery, antibiotic use or type of the oncological treatment are significant risk factors.

High ligature of the inferior mesenteric artery is used by many authors as standard care during rectal or rectosigmoideal resection, as a part of lymphadenectomy, or due to necessity of left bowel mobilization. The ligature of the inferior mesenteric artery worsened the vascularization of the proximal part of the anastomosis, thus requiring the evaluation of the level of proximal bowel resection (18). Due to this reason, the control of blood pressure by Doppler sonography is advised (19). Another condition is the appropriate mobilization of the bowel, which is essential for the performance of tension-free suture.

Both antibiotics and oncological therapy respresent factors, that were not found to be high risk; many authors do not prefer the systemic use of antibiotics (15). In oncological therapy, we can find many factors that can be alternatively recognized as high-risk or low-risk (17).

Some papers state that male gender, hypoalbuminaemia or the absence of small pelvis drainage are risk factors. Other papers mention chronic obstructive pulmonary disease, smoking, alcohol abuse or obesity. These factors are mentioned to play a role in ASA status, worsen postoperative morbidity, and thus having a negative impact on the healing of anastomosis.

In conclusion, we can state that among surgeons, there is no consensus in the technique of anastomosis construction just as well as in the potential risk factors. Nevertheless, we can state that a majority of authors prefer stapler anastomosis as the one with lowest risk, mainly in the distal part of the anastomosis. A majority of authors agree that age, level of anastomosis, peritonitis and corticosteroids are high-risk factors. These findings correlate with the results of our study.

\section{References}

1. Graham MF, Drucker DE, Diegelmann RF, Elson CO. Collagen synthesis by human intestinal smooth muscle cells in culture. Gastroenterology 1987; 92: 400-405.

2. Ahrendt GM, Tantry US, Barbul A. Intra-abdominal sepsis impairs colonic reoperative collagen synthesis. Am J Surg 1996; 171: 102-108.

3. Furst MB, Stromberg BV, Batchford GJ et al. Bursting strength after corticosteroid treatment. Dis Colon Rectum 1994; 37: 12-15.

4. Tadros T, Wobbes T, Hendricks T. Blood transfusion impairs the healing of experimental intestinal anastomoses. Ann Surg 1992; 215: 276-281.

5. Tadros T, Wobbes T, Hendricks T. Opposite effects of interleukin-2 on normal and transfusion suppressed healing of experimental intestinal anastomoses. Ann Surg 1993; 218: 800-808.

6. Everett WG. A comparsion of one layer and two layer techniques for colorectal anastomosis. Br J Surg 1975; 62: 135-140. 
7. Metheson NA, Irving AD. Single layer anastomosis after rectosigmoid resection. Br J Surg 1975; 62: 239-242.

8. Golligher JC, Simpkins KC, Lintott DJ. A controlled comparsion of one and two layer techniques of suture for high and low colorectal anastomoses. Br J Surg 1977; 64: 609.

9. Beart RW, Kelly KA. Randomized prospective evaluation of the EEA stapler for colorectal anastomoses. Am J Surg 1981; 141: 143-147.

10. Reiling RB, Reiling WA, Bernie WA et al. Prospective controlled study of gastrointestinal stapled anastomoses. Am J Surg 1980; 139: 147152.

11. Dziki AJ, Duncan MD, Harmon JW et al. Advantages of handsewn over stapled bowel anastomosis. Dis Colon Rectum 1991; 34: 442-448.

12. Friend PJ, Scott R, Everett WG et al. Stapling or suturing for anastomoses of the left side of the large intestine. Surg Gynecol Obstet 1990; 170: 373-376.

13. Santos JCM, Batista J, Sirimarco MT et al. Prospective Randomized trial of mechanical bowel preparation in patiens undergoing elective colorectal surgery. Br J Surg 1994; 81: 1673-1676.

14. Burke P, Mealy K, Gellen P et al. Requirement for bowel preparation in colorectal surgery. Br J Surg 1994; 81: 907-910.

15. Stellato TA, Danziger LH, Gordon $\mathbf{N}$ et al. Antibiotics in elective colon surgery. A randomized trial of oral/systemic antibiotik for prophylaxis, Am J Surg 1990; 56: 251-254.

16. Irvin TT, Goligher JC. Ethiology of disruption of intestinal anastomoses. Br J Surg 1973; 60: 461-464.
17. Schrock TR, Deveney CW, Dunphy JE. Factors contributing to leakage of colonic anastomoses. Ann Surg 1973; 177: 513-518.

18. Heald RJ, Leicester RJ. The low stapled anastomosis. Dis Colon Rectum 1981; 24: 437-444.

19. Ambrosetti P, Robert J, Mathew $P$ et al. Left-sided colon and colorectal anastomoses: Doppler ultrasound as an aid to asses bowel vascularization. A prospective evaluation of 200 consecutive cases. Int J Colorectal Dis 1994; 9: 211-214.

20. Mengual-Ballester M, García-Marín JA, Pellicer-Franco E, Guillén-Paredes MP, García-García ML, Cases-Baldó MJ, Aguayo-Albasini JL. Protective ileostomy: complications and mortality associated with its closure. Rev Esp Enferm Dig 2012; 104 (7): 350-354.

21. Sajid MS, Siddiqui MR, Baig MK. Single layer versus double layer suture anastomosis of the gastrointestinal tract. Cochrane Database Syst Rev 2012; 1: CD005477.

22. Neutzling CB, Lustosa SA, Proenca IM, da Silva EM, Matos D. Stapled versus handsewn methods for colorectal anastomosis surgery. Cochrane Database Syst Rev 2012; 2: CD003144.

23. Choy PY, Bissett IP, Docherty JG, Parry BR, Merrie A, Fitzgerald A. Stapled versus handsewn methods for ileocolic anastomoses. Cochrane Database Syst Rev 2011; (9): CD004320.

24. Stumpf M, Junge K, Wendlandt M, Krones C, Ulmer F, Klinge U. Risk factors for anastomotic leakage after colorectal surgery. Zbl Chir 2009; 134 (3): 242-248.

Received May 29, 2013. Accepted April 15, 2014. 